4.46), and inconsistent condom use (OR: 2.23; 95\% CI: 1.05 , 4.75).

Conclusion MSM in Jamaica report high prevalence of STI and HIV. STI testing rates are suboptimal and associated with complex multi-level (socio-demographic, relationship, community) factors. Lifetime history of STI was associated with HIV co-infection, structural factors (unemployment), and sexual practices (inconsistent condom use). Results can inform multilevel STI prevention and care programs tailored for MSM in Jamaica.

\section{P4.20 FACTORS ASSOCIATED WITH OPTING IN FOR CLINIC- BASED SYPHILIS TESTING AMONG TRANSGENDER WOMEN IN JAMAICA}

${ }^{1}$ Carmen Logie, ${ }^{2}$ Kathleen Kenny, ${ }^{3}$ Peter A Newman, ${ }^{4}$ Kandasi Levermore, ${ }^{5}$ Nicolette Jones, ${ }^{4}$ Ava Neil, ${ }^{4}$ Tyrone Ellis, ${ }^{6}$ Annecka Marshall. ${ }^{1}$ University of Toronto, Toronto, Canada, Canada; ${ }^{2}$ University of North Carolina, Chapel Hill, Chapel Hill, US; ${ }^{3}$ University of Toronto, Toronto, Canada; ${ }^{4}$ Jamaica AIDS Support for Life, Kingston - Jamaica; ${ }^{5}$ Jamaica AIDS Support for Life, Kingston, Canada; ${ }^{6}$ University of West Indies, Mona Campus, Kingston Jamaica

\subsection{6/sextrans-2017-053264.517}

Introduction Transgender women experience high sexually transmitted infection (STI) rates, yet there is a lack of information about STI testing uptake among transgender women in low and middle-income countries. We conducted a tablet-based survey to assess syphilis testing uptake and prevalence among transgender women in Jamaica.

Methods We conducted a cross-sectional survey with a peerdriven recruitment sample of transgender women in Kingston and Ocho Rios, Jamaica. Participants were provided with a coupon with their survey identification (ID) code for voluntary, free, rapid serological syphilis testing. Coupon ID codes for testing uptake/results were linked with survey results. We conducted backwards stepwise logistic regression to determine factors associated with opting in for syphilis testing.

Results Among 137 participants (mean age: 24.0 [SD: 4.5]), $60.6 \%$ opted in for syphilis testing and $10.6 \%$ tested positive. One-quarter (25.2\%) self-reported being HIV-positive; all participants with syphilis infection were HIV-positive. In univariable analyses having multiple partners was associated with reduced odds of opting in for testing (OR: 0.19; 95\% CI: 0.06-0.60). In multivariable analyses controlling for relationship status, HIV-positive participants were four-fold more likely to opt-in for syphilis testing (Adjusted Odds Ratio [AOR: 4.33]; 95\% CI: 1.31-14.26) than HIV-negative participants. Perceived STI risk (AOR: 1.58; 95\% CI: 1.04-2.40) and childhood sexual abuse history (AOR: 2.80; 95\% CI: 1.03-7.62) were associated with increased odds of opting in for testing. Incarceration history (AOR: 0.27; 95\% CI: 0.110.71 ) was associated with reduced odds of opting in for syphilis testing.

Conclusion Transgender women in Jamaica experience high HIV and syphilis prevalence, and syphilis and HIV co-infection. Findings suggest opt-in clinic based syphilis testing may miss the opportunity to provide testing for some transgender women at elevated STI risk. Future research should assess whether point-of-care syphilis testing may increase testing uptake.

\section{P4.21 SIDE CHICKS, THOTS AND SIDE DICKS: TYPOLOGIES AND DRIVERS OF CONCURRENT PARTNERSHIPS AMONG PEOPLE OF BLACK CARIBBEAN ETHNICITY IN ENGLAND AND IMPLICATIONS FOR STI PREVENTION AND PARTNER NOTIFICATION}

Cath Mercer, ${ }^{1,2} \mathrm{~S}$ Wayal, ${ }^{1,2}$ Gilbart VI, 1,2 E Garnett, ${ }^{1,2}$ Sutcliff Lj, 1,3 P Weatherburn, ${ }^{1,4} \mathrm{G}$ Hughes. 'National Institute of Health Research (NIHR) Health Protection Research Unit (HPRU) in Blood Borne and Sexually Transmitted Infections, UK; ${ }^{2}$ Centre for Sexual Health and HIV, Research Department of Infection and Population Health, University College London, UK; 3 London School of Hygiene and Tropical Medicine, London, UK; ${ }^{4}$ Public Health UK

\subsection{6/sextrans-2017-053264.518}

Introduction In Britain, STI diagnoses rates are highest among black Caribbeans compared to other ethnic groups. The prevalence of concurrency is also high in this population. Concurrent partnerships, (i.e. having sexual partners overlapping in time), can enhance the rate and speed of STI transmission. We explored typologies and drivers of concurrency in black Caribbeans in England and considered their implications for STI prevention.

Methods Using purposive sampling, we recruited people of black Caribbean ethnicity aged $\geq 15$ years from community settings and STI clinics. Audio-recorded 4 focus group discussions ( $\mathrm{n}=28$ participants) and 24 in-depth interviews were conducted between June 2014-Dec 2015 using topic guides. Data was transcribed, managed using NVivo software and analysed using thematic framework to identify patterns of concurrent partnerships and condom use, and reasons for concurrency.

Results 32 women and 20 men (age range: 15-70 years) identifying as heterosexual participated. Open, situational, and experimental concurrent partnerships were commonly reported. Open concurrent partnerships involved a person having a main sexual partner and, in the case of men, additionally having sex with other "side chicks" or "thots", and in the case of women, "side dicks". Situational partnerships involved having a sexual relationship with an ex-partner, especially with someone with whom they had had a child, while having another main sexual partner. These types of partnerships were usually long-term, and condoms were less likely to be used due to emotional attachment, if a co-parent was single and then condomless sex was perceived as a way to "entice" the partner back, or due to the relationship being founded on sexual pleasure. Usuallypeople were aware of the concurrent nature of these partnerships. Experimental concurrent partnerships, commonly reported by single participants, were usually short term, and mostly involved condom use. These were fuelled by lack of readiness to settle with a single partner, or trying to figure out the type of partner they may want to settle with. Other commonly reported reasons for concurrency were low self-esteem at an individual level. At a socio-cultural level, although concurrency was frowned upon, it was perceived as "a Black Caribbean thing" with references being 